\title{
Parâmetros hematológicos e bioquímicos de cabras lactantes alimentadas com dietas contendo glicerina bruta oriunda da produção de biodiesel proveniente de óleo de fritura
}

\author{
[Hematological and biochemical parameters of lactating goats fed diets containing crude glycerin \\ from biodiesel production from waste frying oil] \\ C.B. Santos ${ }^{1}$, M.J. Araújo ${ }^{2 *}$, L.R. Bezerra ${ }^{3}$, C.A.T. Marques $^{4}$, J.N.C. Torreão ${ }^{5}$, \\ N.E. Freitas ${ }^{1}$, C.B. Oliveira Neto ${ }^{6}$, J.S. Morais $^{7}$ \\ ${ }^{1}$ Aluno de pós-graduação - Universidade Federal do Piauí - Bom Jesus, PI \\ ${ }^{2}$ Universidade Federal do Piauí - Bom Jesus, PI \\ ${ }^{3}$ Universidade Federal de Campina Grande - Patos, PB \\ ${ }^{4}$ Universidade Federal de Sergipe - Nossa Senhora da Glória, SE \\ ${ }^{5}$ Instituto Federal de Sergipe - Aracaju, SE \\ ${ }^{6}$ Aluno de graduação - Universidade Federal do Piauí - Bom Jesus, PI \\ ${ }^{7}$ Aluno de pós-graduação - Universidade Federal Rural de Pernambuco - Recife, PE
}

\section{RESUMO}

Objetivou-se analisar os efeitos da inclusão da glicerina bruta (GB), oriunda da produção de biodiesel proveniente de óleos residuais de fritura, sobre o consumo, e os parâmetros hematológicos e bioquímicos em cabras em lactação. Oito cabras com $42,06 \pm 3,5 \mathrm{~kg}$ de peso corporal foram utilizadas, recebendo dietas contendo $0,7,14$ e $21 \%$ de GB com base na MS total da dieta. O experimento foi conduzido em quadrado latino duplo $4 \times 4$, com duração de 80 dias, sendo quatro períodos de 20 dias, dos quais 15 para a adaptação e cinco para a coleta de dados. As amostras de sangue foram coletadas pela punção da veia jugular. Os consumos de MS, proteína bruta e matéria mineral decresceram linearmente, enquanto o de extrato etéreo aumentou quadraticamente. As concentrações do hematócrito, hemoglobina, hemácias e fósforo reduziram linearmente. Tendência quadrática foi observada para as concentrações séricas de cálcio. Não foram verificados efeitos para as concentrações de magnésio, glicose, colesterol total, triglicerídeos, proteínas plasmáticas totais, albumina, globulinas, ureia, creatinina, aspartato aminotransferase e fosfatase alcalina. Conclui-se que a GB pode ser utilizada em até 7\% da MS total da dieta, sem promover problemas metabólicos.

Palavras-chave: coproduto, glicerol, hemoglobina, hemograma

\begin{abstract}
The aim of this study was to analyze the effects of the inclusion of crude glycerin (CG) from biodiesel production from waste frying oils on nutrients intake, hematological and biochemical parameters in lactating goats. Eight goats with $42.06 \pm 3.5 \mathrm{~kg}$ of body weight were used, receiving diets containing 0,7 , 14 and $21 \%$ of CG based on total dietary DM. The experiment was carried out in a $4 \times 4$ double Latin square, with a duration of 80 days, four periods of 20 days, of which 15 days were for adaptation and five days for data collection. Blood samples were collected by puncture of the jugular vein. Dry matter, crude protein, and mineral matter intakes decreased linearly, while intake of ether extract increased quadratically. The concentrations of hematocrit, hemoglobin, red blood cells, and phosphorus reduced linearly. A quadratic tendency for serum calcium concentrations was observed. No effects were observed for concentrations of magnesium, glucose, total cholesterol, triglycerides, total plasmatic protein, albumin, globulins, urea, creatinine, aspartate aminotransferase, and alkaline phosphatase. It can be concluded that $C G$ can be used in up to $7 \%$ of total dietary DM without promoting metabolic problems.
\end{abstract}

Keywords: blood count, co-product, glycerol, hemoglobin

Recebido em 18 de maio de 2017

Aceito em 17 de janeiro de 2018

*Autor para correspondência (corresponding author)

E-mail: jacome@ufpi.edu.br 


\section{INTRODUÇÃO}

Existe uma preocupação mundial com o meio ambiente em relação aos produtos de origem vegetal que são desenvolvidos para gerar energia. O problema é que muitos desses produtos são desperdiçados e, muitas vezes, são depositados em rios ou rede de esgoto, afetando negativamente o meio ambiente. Nesse sentido, é necessário que se adotem tecnologias que contribuam para o desenvolvimento socioeconômico local e mundial, visando diminuir os impactos negativos gerados no meio ambiente, além de fornecer subsídios que possam ser incluídos na alimentação animal, a fim de proporcionar maior aporte energético e menor custo de produção.

Têm-se buscado alimentos alternativos que possam contribuir para a viabilidade dos custos de produção e, principalmente, para o aumento da eficiência alimentar sem afetar o desempenho produtivo e sem provocar danos à saúde animal. Os óleos de fritura são resíduos da indústria alimentícia que podem ser usados para a produção de biodiesel, dando origem à glicerina bruta. Esta, por sua vez, é fonte de glicerol com potencial de uso na produção animal, substituindo parcial ou totalmente o milho (Martins et al., 2013; Silva et al., 2013; Lage et al., 2014).

A inclusão da glicerina bruta como fonte de glicerol na dieta de cabras lactantes poderá promover alterações no hemograma e na bioquímica sérica, uma vez que situações adversas, como estresse em confinamento e desafios nutricionais impostos aos animais, promovem modificações no metabolismo animal para manter suas funções fisiológicas em completa homeostase. Se as modificações forem superiores à capacidade do organismo em se manter em equilíbrio, as doenças da produção podem ser evidenciadas, causando prejuízos à saúde animal e prejuízos econômicos aos produtores (Silva et al., 2014). Exames laboratoriais de sangue, como hemograma e bioquímica sérica, são necessários para avaliar tal efeito no metabolismo. Nesse sentido, os indicadores sanguíneos permitem analiticamente o reconhecimento de respostas metabólicas, o que possibilita a otimização de pesquisas em nutrição animal (Caldeira et al., 2007).
Mediante o exposto, este trabalho foi realizado com o objetivo de analisar os efeitos da inclusão de glicerina bruta oriunda da produção de biodiesel proveniente de óleos residuais de fritura, com elevado teor de ácidos graxos, em dietas para cabras lactantes, sobre o consumo e os parâmetros hematológicos e bioquímicos.

\section{MATERIAL E MÉTODOS}

O experimento foi conduzido na Universidade Federal do Piauí (UFPI), no município de Bom Jesus, PI, após aprovação pelo Comitê de Ética em Experimentação Animal da UFPI, sob o número de protocolo 037/14. Foram utilizadas oito cabras da raça Anglonubiana, multíparas, com peso corporal (PC) médio de $42,06 \pm 3,5 \mathrm{~kg}$, com idade média de dois anos, estando aos $51 \pm 4$ dias de lactação e clinicamente saudáveis. Os animais foram previamente vermifugados com disofenol 20\% (Ibasa $^{\circledR}$, São Paulo, Brasil), mantidos em regime de confinamento em um galpão coberto com telhas de barro, baias individuais $\left(2 \mathrm{~m}^{2}\right)$ feitas de telas de aço, com piso cimentado, providas de comedouro e bebedouro individuais.

O experimento teve duração de 80 dias e foi composto por quatro períodos de 20 dias. Os primeiros 15 dias de cada período foram utilizados para adaptação dos animais às dietas experimentais, e os cinco dias seguintes destinaram-se à coleta dos dados.

Os tratamentos consistiram em rações completas com níveis crescentes de glicerina bruta, nas proporções de $0,7,14$ e $21 \%$ na MS total da dieta (Tab. 1). O volumoso utilizado foi silagem de milho, na proporção de $50 \%$. A glicerina bruta (GB) utilizada foi oriunda da produção de biodiesel proveniente de óleos vegetais de frituras, incorporada manualmente e homogeneizada ao concentrado, sendo analisada para matéria seca (MS), proteína bruta $(\mathrm{PB}) \mathrm{e}$ extrato etéreo (EE) segundo AOAC (Official..., 1990); glicerol segundo USP (Official..., 2015), energia bruta (EB) em bomba calorimétrica (Parr Instrument Co., Moline, IL), metanol segundo USP (Official..., 2015) e ácidos graxos (Official..., 1990). A GB apresentou a seguinte composição (\% na MS): $57,24 \%$ de MS; $0,75 \%$ de matéria mineral (MM); $0,91 \%$ de PB; $30,62 \%$ de glicerol; $0,16 \%$ de $\mathrm{Na} ; 0,11 \%$ de metanol; $3.787 \mathrm{kcal} / \mathrm{kg} \mathrm{MS}$ de EB; $7,41 \%$ de ácidos graxos 
saturados; $12,95 \%$ de ácidos graxos poliinsaturados e 9,07\% de ácidos graxos monoinsaturados.
As dietas foram formuladas de acordo com as recomendações do NRC (Nutrient..., 2007) para cabras em lactação com produção de $2,0 \mathrm{~kg} /$ dia e $4 \%$ de gordura. Os animais foram alimentados logo após as ordenhas, às oito e às 16 horas.

Tabela 1. Participação dos ingredientes e composição química das dietas experimentais

\begin{tabular}{|c|c|c|c|c|}
\hline \multirow{2}{*}{ Item } & \multicolumn{4}{|c|}{ Glicerina bruta (\% MS) } \\
\hline & 0 & 7 & 14 & 21 \\
\hline \multicolumn{5}{|l|}{ Ingrediente (\%MS) } \\
\hline Silagem de milho & 50,00 & 50,00 & 50,00 & 50,00 \\
\hline Milho moído & 36,50 & 29,50 & 21,02 & 13,00 \\
\hline Farelo de soja & 10,50 & 10,22 & 11,68 & 12,60 \\
\hline Ureia & 0,00 & 0,28 & 0,30 & 0,40 \\
\hline Suplemento mineral* & 2,00 & 2,00 & 2,00 & 2,00 \\
\hline Calcário calcítico & 1,00 & 1,00 & 1,00 & 1,00 \\
\hline Glicerina bruta & 0,00 & 7,00 & 14,00 & 21,00 \\
\hline \multicolumn{5}{|c|}{ Composição química (\%MS) } \\
\hline Matéria seca (como fornecido) & 50,74 & 49,69 & 48,68 & 47,71 \\
\hline Proteína bruta & 13,34 & 13,36 & 13,35 & 13,34 \\
\hline Fibra em detergente neutro & 38,20 & 36,44 & 34,58 & 32,75 \\
\hline Fibra em detergente ácido & 18,73 & 18,29 & 17,94 & 17,56 \\
\hline Extrato etéreo & 3,74 & 5,22 & 6,67 & 8,13 \\
\hline Matéria mineral & 5,65 & 5,59 & 5,61 & 5,60 \\
\hline Carboidratos não fibrosos & 39,06 & 39,38 & 39,78 & 40,16 \\
\hline
\end{tabular}

"Composição (por kg do produto): vitamina $\mathrm{A}=135.000 \mathrm{UI}$; vitamina $\mathrm{D} 3=68.000 \mathrm{UI}$; vitamina $\mathrm{E}=450 \mathrm{UI}$; cálcio = $240 \mathrm{~g}$; fósforo $=71 \mathrm{~g}$; potássio $=28,2 \mathrm{~g}$; enxofre $=20 \mathrm{~g}$; magnésio $=20 \mathrm{~g} ;$ cobre $=400 \mathrm{mg} ;$ cobalto $=30 \mathrm{mg} ;$ cromo $=$ $10 \mathrm{mg}$; ferro $=250 \mathrm{mg}$; iodo $=40 \mathrm{mg} ;$ manganês $=1.350 \mathrm{mg}$; selênio $=15 \mathrm{mg}$; zinco $=1.700 \mathrm{mg}$; flúor $($ máx. $)=710 \mathrm{mg}$.

Os alimentos, as rações e as sobras foram analisados para MS, PB e EE de acordo com a AOAC (Official..., 1990). A determinação da fibra em detergente neutro (FDN) e a da fibra em detergente ácido (FDA) foram realizadas segundo Van Soest et al. (1991), e os carboidratos não fibrosos conforme Hall (2000).

Os animais foram alimentados à vontade, e os consumos de MS, PB, EE e MM foram obtidos por meio do registro do alimento oferecido e das sobras, realizado durante o período de coleta. As sobras foram pesadas sempre pela manhã em sua totalidade, e $30 \%$ foram amostradas; em seguida, acondicionadas em sacos de plásticos, identificadas e congeladas a $-20^{\circ} \mathrm{C}$. Ao final de cada período, as amostras foram descongeladas, homogeneizadas, sendo retirada uma amostra composta, para cada animal, de aproximadamente $250 \mathrm{~g}$, pré-secas em estufa com ventilação forçada a $55^{\circ} \mathrm{C}$ por 72 horas e moídas em um moinho tipo Willey com peneiras de malha de um milímetro.
As amostras de sangue foram coletadas no primeiro, terceiro e quinto dias de coleta, antes da primeira refeição do dia, por punção da veia jugular, para realização do hemograma e do perfil bioquímico. Os tubos utilizados para as coletas de sangue, destinados à análise do hemograma continham anticoagulante (EDTA), e os usados para as análises bioquímicas foram sem anticoagulante, exceto os utilizados para análise da glicose plasmática, que continham fluoreto de sódio. Estes foram centrifugados a 4000rpm por 15 minutos, para separação do soro, sendo acondicionado em tubos tipo "Eppendorf" para posteriores análises bioquímicas. Para a determinação do volume globular (VG), utilizouse a técnica do micro-hematócrito. Capilares de vidro foram preenchidos com $75 \%$ de sangue total, devidamente vedados e centrifugados a 15.000rpm, durante cinco minutos (Birgel, 1982). Após a centrifugação dos capilares, a coluna formada pelas hemácias foi interpretada.

A determinação da concentração de hemoglobina $(\mathrm{Hgb})$ sanguínea foi realizada pelo método do 
cianometa-hemoglobina, por meio da diluição da amostra de sangue total, na proporção de 1:250 em líquido de Drabkin, e a leitura da reação colorimétrica foi realizada em espectrofotômetro, sendo o valor da hemoglobina determinado em g/dL (Birgel, 1982). Para a determinação do número total de hemácias (He), as amostras de sangue foram diluídas, na proporção 1:200, em pipeta hematimétrica com líquido de Gower, e a contagem foi realizada em câmara hematimétrica de Neubauer, modificada segundo Birgel (1982), sendo $\mathrm{o}$ fator apresentado em número de hemácias/mL de sangue. $\mathrm{O}$ volume corpuscular médio (VCM) e a concentração hemoglobínica corpuscular média (CHCM) foram calculados com base nos resultados obtidos para VG, Hgb e He, utilizando-se equações matemáticas (Birgel, 1982).

A contagem do número total de leucócitos foi realizada em Câmara de Neubauer, sendo as amostras de sangue diluídas, na proporção de 1:20, utilizando-se como diluente o líquido de Turk. Com o sangue in natura, foram confeccionados dois esfregaços destinados à contagem diferencial de leucócitos. E, após secagem ao ar, foram corados com panótico rápido (Laborclin ${ }^{\circledR}$ Ltda., Pinhais, Paraná, Brasil). A contagem diferencial foi realizada no aumento de $1000 \times$, sendo contados 100 leucócitos, que foram classificados em microscópio óptico, de acordo com suas características morfológicas e tintoriais, em neutrófilos segmentados, eosinófilos, linfócitos e monócitos.

As concentrações de glicose, colesterol total, triglicerídeos e ureia foram determinadas por colorimetria, com o uso de kits comerciais (Labtest Diagnóstica SA ${ }^{\circledR}$ ). Para determinação da creatinina, foi empregada a técnica cinética enzimática, enquanto as proteínas totais (PT) e albumina (A) foram determinadas pelos métodos do biureto e verde bromocresol, respectivamente. As globulinas $(G)$ foram obtidas da diferença entre PT e albumina. As atividades das enzimas aspartato aminotransferase (AST) e fosfatase alcalina (FA) foram analisadas pelo método enzimático colorimétrico, utilizando-se kits comerciais (Labtest Diagnóstica $\mathrm{SA}^{\circledR}$ ), bem como as análises de cálcio, fósforo e magnésio. As análises foram realizadas em analisador bioquímico semiautomático (Spectrum $^{\circledR}$ Marca Médica, Brasil).

O delineamento experimental utilizado foi o de quadrado latino duplo $(4 \times 4)$, sendo quatro animais, quatro períodos e quatro níveis de inclusão da glicerina bruta (GB). Foram utilizados dois quadrados simultâneos. Todos os dados foram analisados usando o procedimento MIXED do SAS (versão 9.0) (SAS Inst. Inc., Cary, NC), incluindo no modelo o nível de GB, o período e a interação nível de $\mathrm{GB} \times$ período como efeitos fixos. $\mathrm{O}$ animal aninhado dentro do tratamento foi considerado como efeito aleatório. Os efeitos de tratamento sobre as variáveis analisadas foram avaliados usando-se contrastes ortogonais para determinar os efeitos linear e quadrático, bem como os efeitos de $0 \%$ de GB na dieta comparados com as médias de todas as dietas contendo GB. Os contrastes foram significativos quando $\mathrm{P}$-value foi $\leq 0,05$. Considerou-se tendência quando foi $\mathrm{P} \leq 0,10$. $\mathrm{O}$ modelo estatístico adotado foi:

$Y_{i j k}=\mu+T_{j}+P_{k}+(T P)_{j k}+A_{i}\left(T_{j}\right)+e_{i j k}$, em que:

$\mathrm{Y}_{\mathrm{ijk}}=$ valor observado para cada característica analisada; $\mu=$ média geral; $\mathrm{T}_{\mathrm{j}}=$ efeito fixo de tratamento $(\% \mathrm{~GB}) ; \mathrm{P}_{\mathrm{k}}=$ efeito fixo de período de coleta; $(\mathrm{TP})_{\mathrm{ik}}=$ efeito fixo da interação dos níveis de $\mathrm{GB}$ e o período; $\mathrm{A}_{\mathrm{i}}=$ efeito aleatório de animal aninhado em nível de tratamento (GB); $\mathrm{e}_{\mathrm{ijk}}=$ erro aleatório associado a cada observação.

\section{RESULTADOS E DISCUSSÃO}

A inclusão da GB na dieta afetou negativamente os consumos de MS (CMS; $\mathrm{P}=0,001$ ), de PB (CPB; $\mathrm{P}=0,0001)$ e de $\mathrm{MM}$ (CMM; $\mathrm{P}=0,002)$, os quais diminuíram linearmente com o aumento do nível de inclusão de GB (Tab. 2). Esse decréscimo aconteceu, principalmente, quando se incluiu a GB acima de $7 \%$ da MS (Tab. 2). O consumo de EE (CEE) foi influenciado de maneira quadrática $(\mathrm{P}=0,0002$; Tab. 2$)$, sendo o máximo consumo de $0,09 \mathrm{~kg} / \mathrm{dia}$ ao nível de $7 \%$ de inclusão da GB. 
Tabela 2. Consumo de nutrientes por cabras lactantes alimentadas com dietas contendo glicerina bruta oriunda da produção de biodiesel proveniente de óleos residuais de fritura

\begin{tabular}{|c|c|c|c|c|c|c|c|c|c|}
\hline \multirow[b]{2}{*}{ Item } & \multicolumn{4}{|c|}{ Glicerina bruta (\% MS) } & \multirow[b]{2}{*}{ EPM } & \multicolumn{4}{|c|}{ P-valor } \\
\hline & 0 & 7 & 14 & 21 & & $\mathrm{~L}$ & $\mathrm{Q}$ & $\begin{array}{c}0 \% \text { vs } \\
\text { GB }^{1}\end{array}$ & $\begin{array}{c}7 \% \text { vs } \\
14 \text { e } 21 \%{ }^{2}\end{array}$ \\
\hline CMS (kg/dia) & 1,75 & 1,85 & 1,30 & 1,02 & 0,09 & 0,001 & 0,19 & 0,03 & 0,0005 \\
\hline CPB (kg/dia) & 0,24 & 0,25 & 0,17 & 0,13 & 0,01 & 0,0001 & 0,17 & 0,03 & 0,0006 \\
\hline CEE (kg/dia) & 0,07 & 0,09 & 0,08 & 0,03 & 0,005 & 0,006 & 0,0002 & 0,50 & 0,006 \\
\hline CMM (kg/dia) & 0,08 & 0,09 & 0,07 & 0,05 & 0,004 & 0,002 & 0,25 & 0,05 & 0,002 \\
\hline
\end{tabular}

$\mathrm{CMS}=$ consumo de matéria seca; $\mathrm{CPB}=$ consumo de proteína bruta; $\mathrm{CEE}=$ consumo de extrato etéreo; $\mathrm{CMM}=$ consumo de matéria mineral. $\mathrm{EPM}=$ erro-padrão da média. ${ }^{1}$ Efeito de $0 \%$ de inclusão de glicerina bruta (GB) versus todas as dietas contendo GB; ${ }^{2}$ efeito de $7 \%$ de inclusão de GB versus as dietas contendo 14 e $21 \%$ de GB; $\mathrm{L}=$ efeito linear; $\mathrm{Q}=$ efeito quadrático.

De modo geral, a redução do CMS deve-se, possivelmente, ao aumento dos ácidos graxos insaturados na dieta, pois o efeito que os lipídeos promovem sobre ele está diretamente relacionado à depressão da fermentação da fibra, bem como à inibição do crescimento microbiano, modificando, assim, a taxa de passagem da dieta pelo trato gastrointestinal do animal (Vargas et $a l .$, 2002). Em adição, a suplementação lipídica maior que $5 \%$ da MS pode comprometer a ingestão, seja pelo mecanismo regulatório que controla a ingestão de alimento, seja pela capacidade limitada dos ruminantes em oxidar ácidos graxos (Palmquist e Mattos, 2006). O decréscimo no CMS resultou também em decréscimo dos consumos de PB e MM. O consumo elevado de EE se justifica pelo alto teor de ácidos graxos presentes na GB, o que pode estar associado a falhas no processo de separação entre biodiesel e glicerol, fato observado principalmente em agroindústrias de biodiesel de pequeno porte.

As concentrações do hematócrito ( $\mathrm{P}=0,002)$, hemoglobina $(\mathrm{P}=0,02)$ e hemácias $(\mathrm{P}=0,0001)$ aumentaram linearmente com a inclusão da GB na dieta, no entanto o VCM e a CHCM não foram influenciados, variando de 17,35 a $18,70 \mathrm{fL}$ e de 34,56 a $35,17 \%$, respectivamente (Tab. 3). As concentrações sanguíneas da série branca também não foram influenciadas pelos tratamentos, assim os valores de leucócitos, neutrófilos, eosinófilos, monócitos e linfócitos variaram de 12261 a $13207 \times 10^{3} / \mu \mathrm{L}, 7979$ a $8866 / \mathrm{mm}^{3}, 153$ a $202 / \mathrm{mm}^{3}, 344$ a $452 / \mathrm{mm}^{3}$ e 3736 a $293 / \mathrm{mm}^{3}$, respectivamente (Tab. 3).
Os valores médios encontrados para hematócrito, hemoglobina, contagem de hemácias, VCM e CHCM encontram-se dentro dos limites fisiológicos para a espécie caprina, que são: 22 a $38 \%, 8$ a $12 \mathrm{~g} / \mathrm{dL}, 8,0$ a $18,0 \times 10^{6} / \mu \mathrm{L}, 16$ a $25 \mathrm{fL}$ e 30 a $36 \%$, respectivamente (Feldman et al., 2000). O aumento nas concentrações de hemácias pode estar relacionado ao metabolismo hepático, sugerindo que a inclusão da GB tenha disponibilizado maior quantidade de precursores para produção dessas células. Como o fígado atua no metabolismo lipídico, pode-se aventar que a inclusão da GB, com alto teor de ácidos graxos, tenha promovido alterações no número de hemácias (Silva et al., 2012; 2014).

Os valores médios de hemoglobina variaram entre 9,76 e 10,59g/dL, considerados valores baixos, quando comparados aos relatados na literatura (Oliveira et al., 2012; Souza et al., 2015). Todavia, esses valores estão dentro dos limites normais para a espécie. Dessa forma, acredita-se que a inclusão de GB não desencadeou alterações no aporte de oxigenação dos pulmões para os diferentes tecidos (Polizopoulou, 2010).

Em valores absolutos, maiores médias encontradas para os leucócitos foram observadas nos tratamentos com $0 \%$ e $14 \%$ de GB, com 13300 e $13205 / \mathrm{mm}^{3}$, respectivamente, estando ligeiramente acima do padrão sugerido na literatura $\left(4000\right.$ a $\left.13000 / \mathrm{mm}^{3}\right)($ Feldman et al., 2000), o que caracteriza leucocitose para os animais que consumiram esses dois tratamentos. No entanto, os animais apresentaram-se, durante todo o experimento, clinicamente saudáveis. 
Tabela 3. Parâmetros hematológicos em cabras lactantes alimentadas com dietas contendo glicerina bruta oriunda da produção de biodiesel proveniente de óleos residuais de fritura

\begin{tabular}{|c|c|c|c|c|c|c|c|c|c|}
\hline & \multicolumn{4}{|c|}{ Glicerina bruta (\%MS) } & \multirow[b]{2}{*}{ EPM } & \multicolumn{4}{|c|}{ P-valor } \\
\hline Item & 0 & 7 & 14 & 21 & & $\mathrm{~L}$ & Q & $\begin{array}{c}0 \% \text { vs } \\
\mathrm{GB}^{1}\end{array}$ & $\begin{array}{c}7 \% \text { vs } \\
14 \mathrm{e} \\
21 \%^{2}\end{array}$ \\
\hline Hematócrito (\%) & 28 & 27 & 30 & 32 & 0,48 & 0,02 & 0,07 & 0,47 & 0,05 \\
\hline $\begin{array}{l}\text { Hemoglobina } \\
\text { (g/dL) }\end{array}$ & 9,88 & 9,76 & 10,30 & 10,59 & 0,13 & 0,02 & 0,27 & 0,40 & 0,12 \\
\hline $\begin{array}{l}\text { Hemácias } \\
\left(\times 10^{6} / \mu \mathrm{L}\right)\end{array}$ & 14,82 & 15,07 & 15,85 & 16,82 & 0,20 & 0,00 & 0,27 & 0,03 & 0,02 \\
\hline VCM (fL) & 18,14 & 17,35 & 18,70 & 17,59 & 0,26 & 0,83 & 0,92 & 0,74 & 0,26 \\
\hline $\mathrm{CHCM}(\%)$ & 34,71 & 35,14 & 34,56 & 35,17 & 0,21 & 0,81 & 0,79 & 0,62 & 0,60 \\
\hline $\begin{array}{l}\text { Leucócitos } \\
\left(/ \mathrm{mm}^{3}\right)\end{array}$ & 13207 & 12261 & 13170 & 12298 & 281,97 & 0,63 & 0,78 & 0,50 & 0,54 \\
\hline $\begin{array}{l}\text { Neutrófilos } \\
\left(/ \mathrm{mm}^{3}\right)\end{array}$ & 8866 & 8230 & 8292 & 7979 & 240,49 & 0,31 & 0,60 & 0,38 & 0,10 \\
\hline $\begin{array}{l}\text { Eosinófilos } \\
\left(/ \mathrm{mm}^{3}\right)\end{array}$ & 153 & 192 & 189 & 202 & 13,27 & 0,29 & 0,64 & 0,18 & 0,92 \\
\hline $\begin{array}{l}\text { Monócitos } \\
\left(/ \mathrm{mm}^{3}\right)\end{array}$ & 452 & 344 & 396 & 375 & 28,74 & 0,34 & 0,32 & 0,30 & 0,66 \\
\hline $\begin{array}{l}\text { Linfócitos } \\
\left(/ \mathrm{mm}^{3}\right)\end{array}$ & 3736 & 3495 & 4293 & 3742 & 181,24 & 0,62 & 0,67 & 0,83 & 0,33 \\
\hline
\end{tabular}

${ }^{1}$ Efeito de $0 \%$ de inclusão de glicerina bruta (GB) versus todas as dietas contendo GB;

${ }^{2}$ efeito de $7 \%$ de inclusão de GB versus as dietas contendo 14 e $21 \%$ de GB; L = efeito linear; Q = efeito quadrático; $\mathrm{EPM}=$ erro-padrão da média; $\mathrm{VCM}=$ volume corpuscular médio; $\mathrm{CHCM}=$ concentração de hemoglobina corpuscular média.

Segundo Jain (1993), a leucocitose pode ser fisiológica, reativa ou proliferativa, sendo mais comumente observada do que a leucopenia. Nesse caso, a leucocitose fisiológica pode ter ocorrido em resposta à neutrofilia provocada pelo estresse ocorrido pela lactação, que, por si só, já é um processo fisiológico estressante para o animal. Segundo Bezerra et al. (2008), a inversão do quadro leucocitário de caprinos com o evoluir da idade, passando de linfocitário para neutrofílico, deve-se não propriamente à influência da idade, mas, sim, reflete as modificações ocorridas com as mudanças fisiológicas, como é o caso de animais gestantes e no puerpério, quando essas mudanças são mais notórias. Os neutrófilos apresentaram-se com valores acima dos valores sugeridos como normais (1200 a $\left.7200 / \mathrm{mm}^{3}\right)$, caracterizando quadro de neutrofilia. Entretanto, esse resultado não indica que as dietas com GB influenciaram tal aumento, uma vez que a dieta controle também apresentou valores elevados. Segundo Garcia-Navarro (2005), a neutrofilia pode ser fisiológica, ou seja, não ter relação com a doença, sendo, portanto, provocada por uma liberação súbita dos neutrófilos no pool marginal, pois essa situação é evidenciada durante a gestação após exercícios prolongados, vômitos ou convulsões, no estresse e durante a taquicardia. Com isso, deduz-se que a inclusão de GB não foi responsável por tais aumentos, pois os valores encontrados nos níveis de $7 \%$ e $21 \%$ de GB apresentaram-se dentro do normal.

Os valores médios de eosinófilos, monócitos e linfócitos mantiveram-se dentro dos valores de referência para a espécie, que são de: 50 a $650 / \mathrm{mm}^{3}$ para eosinófilos, 0 a $550 / \mathrm{mm}^{3}$ para monócitos e 2000 a $9000 / \mathrm{mm}^{3}$ para linfócitos (Feldman et al., 2000). Embora tenham sido observadas oscilações referentes às médias encontradas para tais parâmetros, a GB não causou efeitos negativos para eles. Esses resultados estão de acordo com os de Silva et al. (2013), os quais estudaram a inclusão de $10 \%$ de GB na MS ( $83 \%$ de glicerol e $0,01 \%$ de metanol) na dieta de bovinos, e não verificaram alteração dos parâmetros do leucograma.

O perfil bioquímico não foi alterado pelos tratamentos, com exceção das concentrações de fósforo, que decresceram linearmente $(\mathrm{P}=0,03)$, 
de cálcio $(\mathrm{P}=0,05)$ e $\mathrm{PT}(\mathrm{P}=0,06)$, que tenderam ao efeito quadrático à medida que se aumentou a inclusão da GB na dieta. Foi em nível de $10 \%$ de
GB que se verificou o maior valor sérico para $\mathrm{Ca}$ $(8,0 \mathrm{mg} / \mathrm{dL})$, e de $9,1 \%$ de GB o maior valor para a PT $(7,28 \mathrm{mg} / \mathrm{dL})$.

Tabela 4. Perfil bioquímico sérico de cabras lactantes alimentadas com dietas contendo glicerina bruta oriunda da produção de biodiesel proveniente de óleos residuais de fritura

\begin{tabular}{|c|c|c|c|c|c|c|c|c|c|}
\hline \multirow{3}{*}{ Item } & \multicolumn{4}{|c|}{ Glicerina bruta (\%MS) } & \multirow{3}{*}{ EPM } & \multicolumn{4}{|c|}{ P-valor } \\
\hline & 0 & 7 & 14 & 21 & & $\mathrm{~L}$ & Q & $0 \%$ & $7 \%$ vs \\
\hline & & & & & & & & $\begin{array}{c}\text { vs } \\
\text { GB }^{1}\end{array}$ & $\begin{array}{c}14 \mathrm{e} \\
21 \%^{2}\end{array}$ \\
\hline Glicose (mg/dL) & 48,21 & 50,89 & 49,89 & 52,34 & 1,23 & 0,50 & 0,99 & 0,53 & 0,93 \\
\hline Colesterol (mg/dL) & 129,99 & 125,03 & 127,79 & 126,90 & 1,53 & 0,99 & 0,65 & 0,51 & 0,60 \\
\hline $\mathrm{TAG}(\mathrm{mg} / \mathrm{dL})$ & 21,73 & 22,15 & 20,82 & 24,46 & 1,00 & 0,27 & 0,36 & 0,76 & 0,80 \\
\hline $\mathrm{PT}(\mathrm{g} / \mathrm{dL})$ & 7,16 & 7,24 & 7,26 & 7,04 & 0,04 & 0,37 & 0,06 & 0,83 & 0,41 \\
\hline Albumina (g/dL) & 3,41 & 3,23 & 3,71 & 3,21 & 0,08 & 0,83 & 0,19 & 0,92 & 0,42 \\
\hline Globulina (g/dL) & 3,75 & 4,01 & 3,56 & 3,83 & 0,09 & 0,75 & 0,99 & 0,86 & 0,34 \\
\hline Relação A:G & 0,91 & 0,81 & 1,04 & 0,84 & 0,08 & 0,45 & 0,65 & 0,87 & 0,76 \\
\hline Ureia $(\mathrm{mg} / \mathrm{dL})$ & 36,12 & 37,24 & 39,43 & 40,08 & 0,96 & 0,19 & 0,84 & 0,34 & 0,40 \\
\hline Creatinina $(\mathrm{mg} / \mathrm{dL})$ & 1,40 & 1,45 & 1,52 & 1,47 & 0,02 & 0,21 & 0,33 & 0,16 & 0,46 \\
\hline Cálcio (mg/dL) & 7,45 & 7,98 & 7,90 & 7,35 & 0,15 & 0,73 & 0,05 & 0,48 & 0,39 \\
\hline Fósforo (mg/dL) & 7,98 & 8,06 & 6,78 & 6,60 & 0,27 & 0,03 & 0,90 & 0,27 & 0,11 \\
\hline Magnésio (mg/dL) & 3,41 & 3,57 & 3,51 & 3,48 & 0,05 & 0,72 & 0,31 & 0,33 & 0,57 \\
\hline $\mathrm{AST}(\mathrm{U} / \mathrm{L})$ & 74,32 & 83,28 & 57,87 & 59,85 & 3,26 & 0,15 & 0,49 & 0,74 & 0,07 \\
\hline FA (U/L) & 356,84 & 353,05 & 351,48 & 347,02 & 1,85 & 0,19 & 0,95 & 0,31 & 0,54 \\
\hline
\end{tabular}

${ }^{1}$ Efeito de $0 \%$ de inclusão de glicerina (GB) versus todas as dietas contendo GB; ${ }^{2}$ efeito de $7 \%$ de inclusão de GB versus as dietas contendo 14 e $21 \%$ de GB; $\mathrm{L}=$ efeito linear; $\mathrm{Q}=$ efeito quadrático. $\mathrm{EPM}=$ erro-padrão da média; $\mathrm{TAG}=$ triglicerídeos; $\mathrm{PT}=$ proteínas totais; $\mathrm{A}=$ albumina; $\mathrm{G}=$ globulina; $\mathrm{AST}=$ aspartato aminotransferase; $\mathrm{FA}=$ fosfatase alcalina.

Em valores absolutos, a concentração plasmática de glicose, nos tratamentos $0 \%(48,21 \mathrm{mg} / \mathrm{dL})$ e $14 \%$ de GB $(49,89 \mathrm{mg} / \mathrm{dL})$, encontra-se ligeiramente abaixo dos valores de referência (50 a $75 \mathrm{mg} / \mathrm{dL}$; Kaneko et al., 2008). Entretanto, os níveis com $7 \%$ e $21 \%$ de GB apresentaram, respectivamente, valores normais $(50,89$ e $52,34 \mathrm{mg} / \mathrm{dL}$ ). Mediante esses resultados, pode-se afirmar que alguns animais apresentaram leve hipoglicemia, embora a glicose plasmática não seja um bom indicador do estado nutricional energético decorrente da insensibilidade da glicemia às mudanças nutricionais e à sua sensibilidade ao estresse. A regulação desse elemento ocorre por meio de mecanismos homeostáticos que são bastante eficientes no organismo, os quais envolvem o controle endócrino por parte da insulina e do glucagon (González et al., 2000).

Apesar de as concentrações de colesterol estarem dentro do padrão recomendado $(80$ a $130 \mathrm{mg} / \mathrm{dL}$; Kaneko et al., 2008), nota-se que, em todos os tratamentos, estiveram sempre próximas ao limite superior. Segundo Villa et al. (2009), as concentrações séricas de colesterol total são consideradas indicadores adequados do total de lipídeos no plasma, pois correspondem a, aproximadamente, $30 \%$ do total e têm uma relação direta com a alimentação do animal. Para os triglicerídeos, os valores médios observados também estiveram no limite inferior do intervalo de referência de triglicerídeos para a espécie caprina (20 a 100mg/dL; Gregory et al., 2009). Isso ocorreu provavelmente devido ao fato de o CMS ter reduzido linearmente e o CEE ter se comportado de forma quadrática, porém esses resultados não foram suficientes para provocar alterações significativas nos indicadores de metabolismo energético dos animais.

As concentrações séricas de PT foram ligeiramente acima dos valores de referência para a espécie caprina $(6,4$ a $7,0 \mathrm{~g} / \mathrm{dL})$, descritos por Kaneko et al. (2008). As concentrações séricas de albumina e globulinas, bem como a relação A:G, não sofreram influências dos tratamentos e mantiveram-se dentro dos valores de referência (2,7 a 3,9g de albumina/dL e 2,7 a 4,1g de globulina/dL; Kaneko et al., 2008). Porém, a 
relação A:G apresentou-se reduzida, comparando-se à literatura. A redução dessa relação pode ser justificada pelos níveis de globulina observados no experimento, o que demonstra que as PT aumentam com a idade, em decorrência da diminuição da albumina e elevação da globulina (Jain, 1993). Com o avançar da lactação, ocorre aumento das globulinas e diminuição da albumina, podendo os valores alcançar uma relação de 1:4, segundo Kaneko et al. (2008), permanecendo, assim, fora do intervalo ideal para a espécie, que é de 1:1. Essa relação $A: G$ alterada, muito provavelmente devido ao período de lactação, pode ter ocasionado a neutrofilia, observada anteriormente, e, consequentemente, a leucocitose, sendo ambas fisiológicas. Em relação aos valores absolutos, não houve influência das dietas com GB. Esses resultados corroboram, em parte, os de Martins et al. (2013), que, quando avaliaram as concentrações séricas de albumina em borregas Texel, suplementadas com $20 \%$ de glicerol, não observaram alteração nas concentrações séricas de albumina.

As concentrações séricas de ureia e creatinina mantiveram-se dentro dos valores de referência $(21,4$ a $42,8 \mathrm{mg} / \mathrm{dL}$ e 1,0 a $2,0 \mathrm{mg} / \mathrm{dL}$, respectivamente; Kaneko et al., 2008), com isso, possivelmente a inclusão da GB não afetou a função renal dos animais (Braun et al., 2010). Em adição, acredita-se que a produção de amônia ruminal manteve-se normal, fazendo com que os teores de ureia sanguínea permanecessem normais. Essa afirmação é suportada em razão da existência de uma correlação positiva entre a ureia sanguínea e a amônia ruminal, bem como do uso de aminoácidos (alanina, glutamina e glicina) precursores gliconeogênicos no fígado (Kozloski, 2011).

Para todos os tratamentos, as concentrações séricas de cálcio estão abaixo dos valores normais para caprinos $(8,9$ a $11,7 \mathrm{mg} / \mathrm{dL}$; Kaneko et al., 2008). Essa tendência pode ser justificada pelo menor CMS, consequentemente, menor CMM verificado neste estudo (Tab. 2). Na prática, percebeu-se que os animais rejeitavam o concentrado com maior participação de GB (14\% e $21 \%$ ), selecionando com mais frequência o volumoso da dieta (silagem de milho), o que pode ter alterado a disponibilidade de cálcio no organismo animal. Segundo Berchielli et al.
(2011), as plantas forrageiras, por si, não são capazes de atender as exigências em minerais dos animais; além disso, conforme os mesmos autores, dietas deficientes reduzem a concentração sanguínea de $\mathrm{Ca}$, estimulando a secreção do paratormônio (PTH), que, por sua vez, estimula a reabsorção óssea por ativação dos osteoclastos. Os resultados observados no presente estudo estão de acordo com os achados de Mundim et al. (2007), ao trabalharem com cabras em lactação, os quais verificaram valores máximo e mínimo de cálcio sérico de 10,30 e $6,40 \mathrm{mg} / \mathrm{dL}$, respectivamente.

As concentrações séricas de fósforo diminuíram à medida que aumentou o nível de inclusão de GB na dieta, entretanto apresentaram-se dentro dos padrões normais (4,2 a 9,1 mg/dL; Kaneko et al., 2008). Observou-se que, à medida que aumentou a oferta energética advinda da GB na dieta (Tab. 1), as concentrações séricas de fósforo diminuíram. Tal fato pode estar relacionado com a participação deste mineral no metabolismo energético, visto que, quanto maior o consumo de substratos energéticos, maior é a quantidade de fósforo gasto no espaço intracelular, reduzindo sua concentração no plasma (Ribeiro et al., 2003). Os mesmos fatores que promovem a assimilação de cálcio atuam no controle da manutenção do fósforo, ou seja, também é regulada pelo paratormônio, pela calcitonina e pela vitamina D (González et al., 2000).

A redução das concentrções de fósforo também pode estar associada à redução do CMS e do CMM. Os valores encontrados no presente estudo são compatíveis com os encontrados por Mundim et al. (2007), que, ao avaliarem o perfil bioquímico sanguíneo de cabras da raça Saanen, encontraram valores mínimos e máximos para fósforo de 3,30 e 12,50mg/dL, respectivamente.

As concentrações séricas de magnésio não foram influenciadas $(\mathrm{P}>0,05)$ pela inclusão da GB na dieta, estando os valores dentro da normalidade (2,8 a 3,6mg/dL; Kaneko et al., 2008). Situações de hipomagnesemia promovem consequências sérias para os ruminantes, podendo levar a óbito. Tal situação ocorre quando existe baixa ingestão de magnésio na dieta e, em casos de excessiva lipólise em decorrência de uma deficiência de energia, pode ocasionar anormalidades digestivas e diminuição da produção de leite. 
As atividades das enzimas AST e FA mantiveram-se dentro dos valores de referência (43 a 132U/L e 93 a 347U/L, respectivamente; Kaneko et al., 2008). Dessa forma, pode-se inferir que a GB $(30,6 \%$ de glicerol e $0,11 \%$ de metanol) não provocou efeitos tóxicos, pois essas enzimas são consideradas biomarcadores sanguíneos de grande valor para avaliar distúrbios metabólicos, funcionamento hepático, alterações ósseas e desequilíbrio na relação $\mathrm{Ca}: \mathrm{P}$ (González et al., 2000). Isso porque a FDA (2006) indica que os teores de metanol superiores a $150 \mathrm{ppm}(0,15 \%)$ podem ser considerados impróprios para a alimentação animal.

\section{CONCLUSÃO}

Conclui-se que a glicerina bruta, como fonte de glicerol, na dieta de cabras lactantes pode ser incluída em até $7 \%$ da matéria seca total da dieta, sem promover problemas metabólicos.

\section{REFERÊNCIAS}

BERCHIELLI, T.T.; PIRES, A.V.; OLIVEIRA, S.G. (Eds.). Nutrição de ruminantes. Jaboticabal: FUNEP, 2011. 616p.

BEZERRA, L.R.; FERREIRA, A.F.; CAMBOIM, E.K.A. et al. Profile hematological of goat clinical healthy servants in Cariri paraibano. Ciênc. Agrotec., v.32, p.955-960, 2008.

BIRGEL, E.H. Hematologia clínica veterinária. In: BIRGEL, E.H.; BENESI, F.J. (Eds.). Patologia clínica veterinária. São Paulo: Sociedade Paulista de Medicina Veterinária, 1982. p.2-34.

BRAUN, J.P.; TRUMEL, C.; BÉZILLE, P. Clinical biochemistry in sheep: a selected review. Small Ruminant Res., v.92, p.10-18, 2010.

CALDEIRA, R.M.; BELO, A.T.; SANTOS, C.C. et al. The effect of long-term feed restriction and over- nutrition on body condition score, blood metabolites and hormonal profiles in ewes. Small Ruminant Res., v.68, p.242-255, 2007.
FOOD AND DRUG ADMINISTRATION. Code of Federal Regulations - CFR, Title 21, 6: 21, CFR582.1320. Disponível em: https://www.accessdata.fda.gov/scripts/cdrh/ cfdocs/cfCFR/CFRSearch.cfm (04-12-2015) FDA, 2006.

FELDMAN, B.F., ZINKL, J.G., JAIN, C.N. Schalm's veterinary hematology. Philadelphia: Lippincott Williams \& Wilkins, 2000. 1344p.

GARCIA-NAVARRO, C.E.K. Manual de hematologia veterinária. São Paulo: Varela, 2005. 206p.

GONZALEZ, F.H.D.; BARCELLOS, J.; PATIÑO, H.O.; RIBEIRO, L.A. Perfil metabólico em ruminantes. Seu uso em nutrição e doenças nutricionais. Porto Alegre: UFRGS, 2000. 108p.

GREGORY, L.; BARDESE, C.B.; BIRGEL JR, E.H. et al. Lipidograma e glicemia de caprinos da raça Saanen, durante os primeiros dias de vida. ARS Vet., v.25, p.109-115, 2009.

HALL, M.B. Neutral detergent-soluble carbohydrates. Nutritional relevance and analysis. Gainesville: University of Florida, 2000.76p.

JAIN, N.C. Essentials of veterinary hematology. Philadelphia: Lea \& Febiger, 1993, 417p.

KANEKO J.J.; HARVEY J.W.; BRUSS, M.L. Clinical biochemistry of domestic animals. Academic Press: San Diego, 2008. 928p.

KOZLOSKI, G.V. Bioquímica dos ruminantes. Santa Maria: UFSM, 2011. 280p.

LAGE, J.F.; PAULINO, P.V.R.; PEREIRA, L.G.R. et al. Carcass characteristics of feedlot lambs fed crude glycerin contaminated with high concentrations of crude fat. Meat Sci., v.96, p.108-113, 2014.

MARTINS, A.S.; KACHINSKI, M.B.; GALETTO, S.L. et al. Consumo, desempenho e metabólitos sanguíneos de ovelhas suplementadas com glicerol na dieta. Encicl. Biosf., v.9, p.788-797, 2013.

MUNDIM, A.V.; COSTA, A.S.; MUNDIM, S.A.P. et al. Influência da ordem e estágio da lactação no perfil bioquímico sanguíneo de cabras da raça Saanen. Arq. Bras. Med. Vet. Zootec., v.59, p.306-312, 2007. 
NUTRIENT of small ruminants: sheep, goats, cervids, and new world camelids. Washington: National Academy Press, 2007. 384p.

OFFICIAL compendia of standards. United States Pharmacopeial Convention. 38.ed. Rockville: USP, 2015.

OFFICIAL methods of analysis. 15.ed. Washington: AOAC, 1990.

OLIVEIRA, M.G.C.; NUNES T.L.; PAIVA, A.L.C. et al. Aspectos hematológicos de caprinos (Capra hircus) da raça Canindé criados no Rio Grande do Norte. Pesqui. Vet. Bras., v.32, p.4-8, 2012.

PALMQUIST, D.L.; MATTOS, W.R.S. Metabolismo de lipídeos. In: BERCHIELLI, T.T.; PIRES, A.V.; OLIVEIRA, S.G. (Ed). Nutrição de ruminantes, Jaboticabal: FUNEP, 2006. p. 287-310.

POLIZOPOULOU, Z.S. Haematological tests in sheep health management. Small Ruminant Res., v.92, p.88-91, 2010.

RIBEIRO, L.A.O.; GONZÁLEZ, F.H.D.; CONCEIÇÃO, T.R. et al. Perfil metabólico de borregas Corriedale em pastagem nativa do Rio Grande do Sul. Acta Sci. Vet., v.31, p.167-170, 2003.

SILVA, D.A.V.; EZEQUIEL, J.M.B.; PASCHOALOTO, J.R.; ALMEIDA, M.T.C. Volumosos e glicerina bruta na dieta de bovinos de corte: efeito sobre o hemograma e bioquímica sérica. ARS Vet., v.29, p.183-189, 2013.
SILVA, D.A.V.; HOMEM JÚNIOR, A.C.; EZEQUIEL, J.M.B. Sexo e fontes de lipídeos sobre os parâmetros sanguíneos de ovinos confinados. Arq. Bras. Med. Vet., v.36, p.153158, 2014.

SILVA, D.A.V.; VAN CLEEF, E.H.C.B.; EZEQUIEL, J.M.B. et al. Glicerina bruta na dieta de bovinos de corte confinados: efeito sobre o hemograma. Braz. J. Vet. Res. Anim. Sci., v.49, p.202-209, 2012.

SOUZA, P.T.; SALLES, M.G.F.; COSTA, A.N.L. et al. Perfil hematológico de cabras Saanen e mestiças ( $1 / 2$ Saanen e $1 / 2$ Anglonubiana) criadas em clima tropical do Ceará. Pesqui. Agropec. Bras., v.35, p.99-104, 2015.

VAN SOEST, P.J.; ROBERTSON, J.B.; LEWIS, B.A. Methods for dietary fiber, neutral detergent fiber, and nonstarch polysaccharides in relation to animal nutrition. J. Dairy Sci., v.74, p.35833597, 1991.

VARGAS, L.H.; LANA, R.P.; JHAM, G.N. et $a l$. Adição de lipídios na ração de vacas leiteiras: parâmetros fermentativos ruminais, produção e composição do leite. Rev. Bras. Zootec., v.31, p.522-529, 2002.

VILLA, N.A.; PULGARÍN, E.F.; TABARES, P.A. et al. Medidas corporales y concentración sérica y folicular de lípidos y glucosa en vacas Brahman fértiles y subfértiles. Pesqui. Agropecu. Bras., v.44, p.1198-1204, 2009. 